\title{
Stress resistance on the example of supramolecular-genetic level of plant development
}

\author{
Ivanova E.A. \\ Ufa Institute of Biology of the Russian Academy of Sciences, UFITS UIB RAS, Moscow, st. Ufa, Russia \\ email: fiona_belobor@mail.ru
}

\begin{abstract}
Supramolecular chemistry arose as the chemistry of assemblies held together by noncovalent interactions. Through the concept of molecular recognition of the selective binding of complementary components that provide the programmed orientation of the growth and development of self-processes, she came to one of the key concepts - the concept of molecular information (passive and active), which studies the storage of information at the molecular level, as well as the reading, transmission and processing of information on supramolecular level.

Further development of supramolecular chemistry - in the direction of the general science of matter carrying information - is added, from the field of chemistry, to the fundamental triad: matter - energy - structure. Supramolecular chemistry is rapidly expanding the boundaries of chemical science to physical, biological and even sociological phenomena. Three main concepts become its foundation: fixation (binding), recognition and coordination.
\end{abstract}

The aim of the work is to present the interphase total chromatin matrix of the cell nucleus in the form of supramolecular structures: Np-nucleoplasm, Chr-I-fragile, Chr-II strongly associated with the nuclear matrix-NM and, in fact, the NM itself, on the interface of which there is a dynamic reorganization of the nuclear supermolecular proteome in a stress environment from the standpoint of epigenetic developmental biology.

The object of the study is spring wheat, bred by breeders, after a long period of cold stress, in winter. The experimental phase of ontogenesis is the initiation of growth morphogenesis. The entire experimental volume of work was carried out on patents developed in the laboratory of mathematical and molecular genetics, where the features of the isolation of cell nuclei, their supramolecular structures and elution from the latter, supermolecular blocks - non-histone and histone proteins are presented.

An algorithm for the dynamics of proteomic ensembles of supermolecular structures on the interface of supramolecular chromatin blocks is shown, on which the positioning role of $(\mathrm{H} 3+\mathrm{H} 4) "$ - arginine-rich histones in stressresistance, in the zone of meristem geomorphism of the root system of a winter cultivar, in the period of initiation of coordinated regular growth and formation of stabilization of the integrative physiological system of the regulating function of the whole organism. The presented data "from genomics to integrative physiology" lead to the concept of an epigenetic polyandrum.

Acknowledgements: The work was carried out within the framework of the state assignment of the Ministry of Education and Science of Russia No. 075-00326-19-00 on the topic No. R\&D AAAA-A18-118022190104-7. The instrument base of the Center for Collective Use "Agidel" of the UFIC RAS was used. 